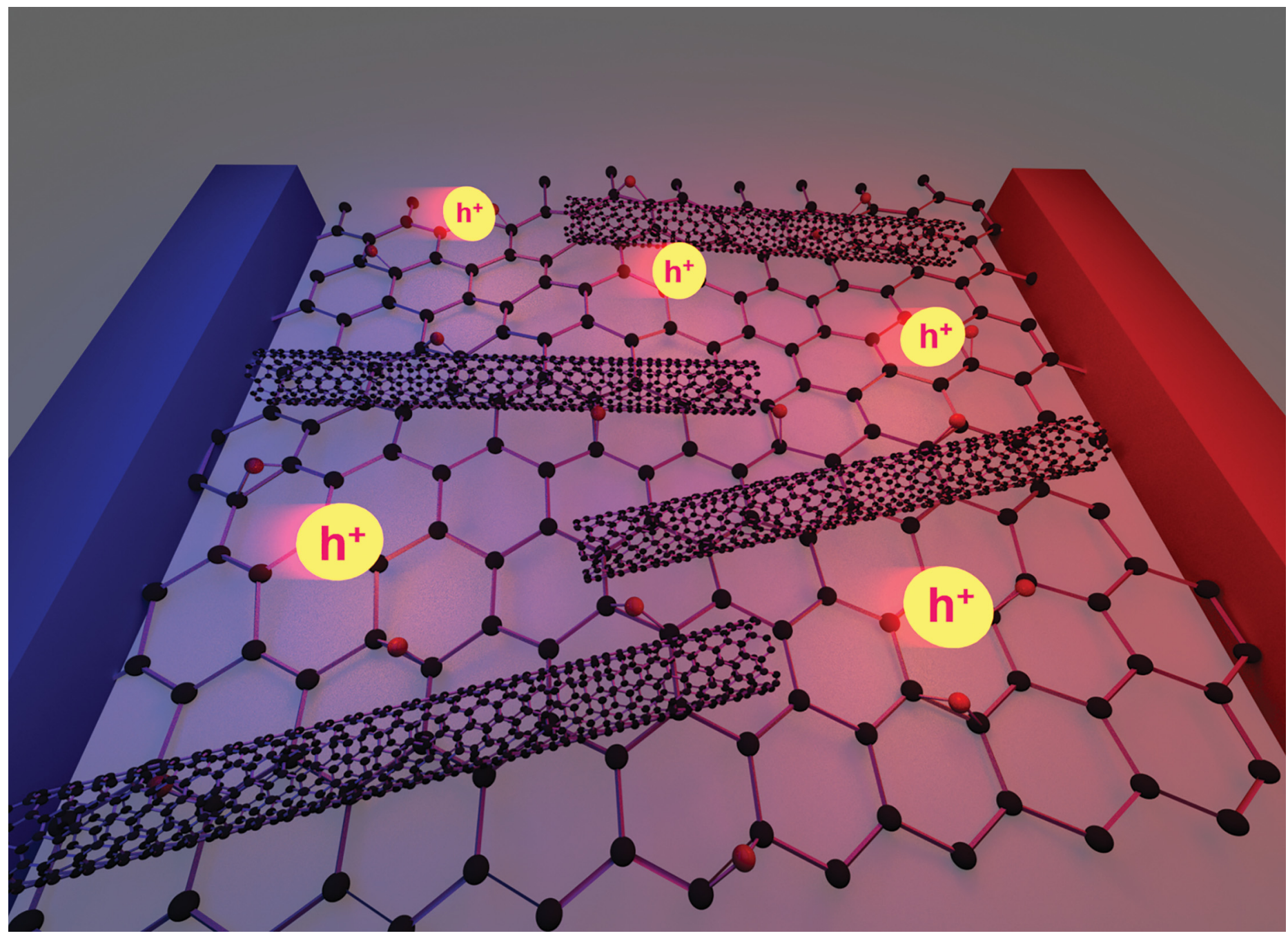

Showcasing research from Professor Shinya Hayami's laboratory, Department of Chemistry, Kumamoto University, Japan.

Enhanced thermoelectric properties exhibited by unreduced freestanding graphene oxide/carbon nanotube membranes

The Seebeck coefficients, electric conductivities, and thermoelectric power factors of a range of unreduced graphene oxide (GO)/single-wall carbon nanotube (CNT) membranes incorporating different GO/CNT ratios as well as of a series of reduced graphene oxide ( $\mathrm{rGO}$ ) membranes for which reduction had occurred at different temperatures have been demonstrated. The considerable thermoelectric power factor of $5.33 \times 10^{-2} \mu \mathrm{W} \mathrm{mK}^{-2}$ observed for unreduced $\mathrm{GO} / \mathrm{CNT}$ (1:2 ratio) implies the suitability of unreduced $\mathrm{GO} / \mathrm{CNT}$ as a flexible thermoelectric material.
As featured in:

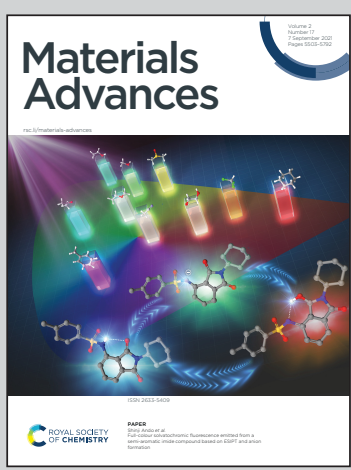

See Shinya Hayami et al., Mater. Adv., 2021, 2, 5645. 
Check for updates

Cite this: Mater. Adv., 2021, 2, 5645

Received 1st April 2021

Accepted 11th June 2021

DOI: 10.1039/d1ma00299f

rsc.li/materials-advances

\title{
Enhanced thermoelectric properties exhibited by unreduced freestanding graphene oxide/carbon nanotube membranes $\dagger$
}

\author{
Md. Saidul Islam, ${ }^{\text {ab }}$ Hitomi Ohmagari, ${ }^{\text {ac }}$ Mohammad Atiqur Rahman, ${ }^{a}$ \\ Yuta Shudo, (D) ${ }^{a}$ Masahiro Fukuda, ${ }^{a}$ Junya Yagyu, ${ }^{a}$ Yoshihiro Sekine, (DD ${ }^{\text {ad }}$ \\ Leonard F. Lindoy (iD ${ }^{e}$ and Shinya Hayami (D) *ab
}

\begin{abstract}
Herein we determine the Seebeck coefficients, electric conductivities, and thermoelectric power factors of a range of unreduced graphene oxide (GO)/single-wall carbon nanotube (CNT) membranes incorporating different GO/CNT ratios as well as of a series of reduced graphene oxide ( $\mathrm{rGO}$ ) membranes for which reduction had occurred at different temperatures. The considerable thermoelectric power factor of $5.33 \times 10^{-2} \mu \mathrm{W} \mathrm{mK}^{-2}$ generated from unreduced GO/CNT (1:2 ratio) is in agreement with its suitability for application as a flexible thermoelectric material. Moreover, compared to the conventional architectures of traditional thermoelectric materials, the inherent flexibility of GO-based thermoelectric materials opens the door to improved performance and efficiency, with lightweight, scalable, stretchable, and/or wearable materials being the outcome.
\end{abstract}

\section{Introduction}

The thermoelectric phenomenon is considered as a green technique to produce electrical energy using the thermal electromotive force induced due to a temperature difference between two ends of a thermoelectric material, where charge carriers diffuse across the temperature gradient. This results in a build-up of charge and thus creates a potential difference. ${ }^{1-4}$ Thermoelectric devices are all-solid-state devices with no moving parts and thus can be employed without generating noise or other pollution. Even though the energy efficiency from thermoelectric process is quite small, the widespread use of low-temperature driven thermoelectric energy conversion devices could be useful for producing electricity anywhere by utilizing the heat from sunlight, the waste heat from different systems or even from the human body. So far, the best thermoelectric materials are

\footnotetext{
${ }^{a}$ Department of Chemistry, Faculty of Advanced Science and Technology, Kumamoto University, 2-39-1 Kurokami, Kumamoto 860-8555, Japan. E-mail: hayami@kumamoto-u.ac.jp

${ }^{b}$ Institute of Industrial Nanomaterials (IINa), Kumamoto University, 2-39-1 Kurokami, Kumamoto 860-8555, Japan

${ }^{c}$ College of Science and Engineering, Aoyama Gakuin University, 5-10-1 Fuchinobe, Chuo-ku, Sagamihara, Kanagawa 252-5258, Japan

${ }^{d}$ Priority Organization for Innovation and Excellence, Kumamoto University, 2-39-1 Kurokami, Chuo-ku, Kumamoto 860-8555, Japan

${ }^{e}$ School of Chemistry F11, The University of Sydney, Sydney, New South Wales, 2006, Australia

$\dagger$ Electronic supplementary information (ESI) available. See DOI: 10.1039/d1ma00299f
}

inorganic compounds (such as $\mathrm{Bi}_{2} \mathrm{Te}_{3}$ ) that have relatively low earth abundance and are fabricated through highly complex vacuum processing routes that limit their full potential for widespread use. Consequentially, the development of alternative efficient, cheap, and abundant thermoelectric materials represents the key to the future advancement and large scale commercialization of thermoelectric devices. ${ }^{4-7}$ The suitability of a thermoelectric material is determined by the device efficiency which is related to the thermoelectric figure of merit $\mathrm{z} T$, given by zT $=\sigma S^{2} T / k$ where $\sigma S, T$, and $k$ represent the material's electrical conductivity, Seebeck coefficient, temperature, and thermal conductivity, respectively. The term $\sigma S^{2}$ corresponding to the power factor of a thermoelectric material, plays a significant role in determining its efficiency. ${ }^{8-10}$

Compared to 3D bulk materials, decreasing the dimension of a thermoelectric compound towards a $2 \mathrm{D}$ structure is expected to result in better thermoelectric properties, including higher electrical conductivity coupled with lower thermal conductivity as a consequence of the discretization of the electric state density, the construction of interfaces between the layered compound, and the quantum confinement effect. ${ }^{11-14}$ In addition, compared to the conventional bulky 3D geometrical architectures of traditional thermoelectric materials, the flexibility in the design of such 2D materials open the possibility for improving performance and giving rise to lightweight, scalable, stretchable, and wearable properties. ${ }^{15-17}$ The unique properties of graphene oxide (GO) sheets along with the uniform layered stacking arrangement with sufficient mechanical strength to act as a free-standing 
membrane is a distinctive advantage making GO attractive for thermoelectric application. Unlike graphene with its high thermal conductivity which limits its suitability for practical thermoelectric applications (despite a considerable Seebeck coefficient and high electrical conductivity), density functional theory (DFT) results indicate that adding oxygen into the graphene lattice (i.e., to produce GO) significantly reduces its thermal conductivity due to the phonon scattering effect resulting from both acoustic mismatch and reduced symmetry in the graphene structure; hence GO appears a promising candidate for constructing thermoelectric materials. ${ }^{18,19}$ Nevertheless, pristine GO is associated with low electric conductivity and this is identified as the key concern for its practical use in GO-based thermoelectric materials. Previous studies on GO-based thermoelectric materials have focused on reduced graphene oxide (rGO) for improving the electrical conductivity while the restoration of some $\mathrm{sp}^{2}$ hybridized $\mathrm{C}$ atoms has been shown to be responsible for improved electrical conductivity. ${ }^{20-23}$ Unfortunately, the Seebeck coefficient is sharply decreased with a high degree of rGO reduction, with the expectation that thermal conductivity will increase due to the graphene-like structure of rGO. Even though, the GO-based hybrid prepared by adding carbon nanotubes (CNT) to the GO has been reported to increase in thermal conductivity which was attributed to the formation of $3 \mathrm{D}$ heat conduction paths by the addition of MWCNTs. ${ }^{24}$ However, the thermal conductivity of $\mathrm{GO} / \mathrm{CNT}$ is expected to the less than that of pristine CNT or rGO and might be facilitated the thermoelectric properties of GO/CNT hybrid.

Thus, in the present work we consider the prospect of employing unreduced GO for use in thermoelectric materials. The electrical conductivity of unreduced GO membrane was increased by the introduction of single wall CNT in different ratios while keeping the functional groups of the GO intact. In addition, we also investigated the effect of the temperature used for the reduction of GO (annealed at different temperatures ranging from 100 to $400{ }^{\circ} \mathrm{C}$ ) on the corresponding thermoelectric behaviour. The Seebeck coefficients and electrical conductivities have been measured and the corresponding thermoelectric power factors $\left(\sigma S^{2}\right)$ calculated. The maximum thermoelectric potential and Seebeck coefficient were observed for rGO whose reduction occurred at $100{ }^{\circ} \mathrm{C}$. However, the highest electric conductivity and thermoelectric power factor were obtained from the sample composed of a $1: 2$ ratio of GO/CNT.

\section{Experimental}

GO dispersion in water was purchased from Nippon Shokubai Co., Ltd. CNT was obtained from Sigma Aldrich. The GO film was prepared from the GO dispersion $(1 \mathrm{mg} / 5 \mathrm{~mL})$ employing a membrane filter with a pore size of $0.4 \mu \mathrm{m}$, followed by drying the product film under ambient conditions. Thermal annealing at the desired temperature was employed to prepare the rGO samples. The products from reduction at 100, 120, 140, 160, 300, $400{ }^{\circ} \mathrm{C}$ were designated as rGO@100-rGO@400, respectively.
Unreduced GO and CNT dispersion were mixed in the ratios (by weight) $1: 0.5,1: 1$ and $1: 2$ and denoted as GO/CNT(1:0.5), $\mathrm{GO} / \mathrm{CNT}(1: 1)$, and GO/CNT(1:2), respectively. The mixed suspensions were each filtered through a membrane using reduced pressure to obtain freestanding GO/CNT membranes.

The structure and morphology of the samples were characterized by field emission scanning electron microscopy (FE-SEM, JSM-7600F, JEOL), Fourier transform infrared spectroscopy (FT-IR, Spectrum Two, PerkinElmer) and X-ray diffraction spectroscopy. Seebeck coefficients for the respective samples involved measuring the temperature differences between opposing ends of individual samples. The dimension including length, width and thickness of each sample were $1.8 \mathrm{~cm}, 0.5 \mathrm{~cm}$ and $\sim 30 \mu \mathrm{m}$, respectively. One end is contacted with a peltier element connected to a power supply (hot end) while the opposite end was maintained at ambient temperature (cold end) to create a temperature difference between these ends as shown in Fig. S1 (ESI $\dagger$ ). The temperature difference was maintained at $36 \mathrm{~K}$. The thermo-voltage generated between the two ends was measured using a PC510 digital multimeter by attaching silver-pasted thin copper wire probes in contact with each end.

\section{Results and discussion}

The SEM images (Fig. 1a and b) show the cross-sectional morphology of the GO and GO/CNTs (1:2) membranes, respectively. Clearly, GO shows a characteristic layer by layer membrane morphology. For the GO/CNT membrane, the CNT is found to be well dispersed in a compact arrangement in the resulting freestanding membrane.

The SEM images of GO/CNT $(1: 0.5)$ and GO/CNT $(1: 1)$ are given in Fig. S2 (ESI $\dagger$ ). Upon reduction of GO membrane at higher temperatures $\left(100\right.$ to $\left.400{ }^{\circ} \mathrm{C}\right)$, the interlayer distance for GO is decreased and this was also confirmed by PXRD. The (a)

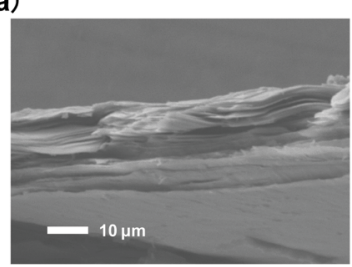

(c)

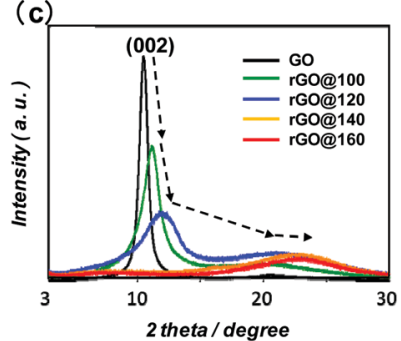

(b)

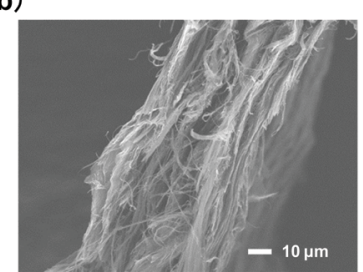

(d)

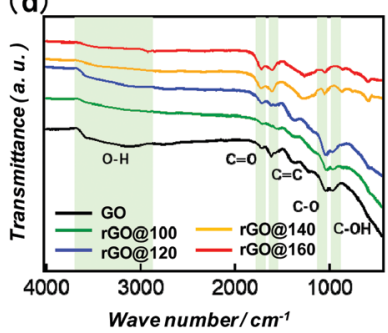

Fig. 1 Cross sectional SEM images. (a) GO and (b) GO/CNT with $1: 2$ weight ratio. (c) PXRD patterns for GO and rGO at 100 to $180{ }^{\circ} \mathrm{C}$; (d) FTIR spectra for $\mathrm{GO}$ and $\mathrm{rGO}$ at 100 to $180^{\circ} \mathrm{C}$. 
PXPD patterns for GO and rGO measured after reduction at temperatures ranging from 100 to $160{ }^{\circ} \mathrm{C}$ are presented in Fig. 1c. The characteristic PXRD peak for GO (002) at $2 \theta=$ $10.48^{\circ}$ shifts to the higher angles of $11.15^{\circ}, 11.94^{\circ}, 22.50^{\circ}$ and $22.90^{\circ}$ for rGO@100, rGO@120, rGO@140, and rGO@160, respectively. The calculated interlayer distances for $\mathrm{GO}$, rGO@100, rGO@120, rGO@140, rGO@160 are 8.44 Å, 7.94 A, $7.41 \AA$, $3.95 \AA$, and $3.88 \AA$, respectively. The decrease in the interlayer distances can be attributed to both the removal of the adsorbed water as well as to decomposition of some of the oxygen functional groups.

The XRD patterns for rGO@300 and rGO@400 are shown in Fig. S3 (ESI $\dagger$ ). The respective broad peaks at $24.04^{\circ}$ and $24.58^{\circ}$, indicate that the (002) plane has shifted towards higher angles, with the corresponding interlayer distances being $3.70 \AA$ and $3.62 \AA$, respectively. The interlayer distance is drastically decreased from rGO@120 (7.41 ̊) to rGO@140 (3.95 ̊) indicating that a large number of oxygen-containing functional groups in GO were removed at $140{ }^{\circ} \mathrm{C}$. The FT-IR spectra of rGO@100, rGO@120, rGO@140, rGO@160 as well as of GO were obtained (Fig. 1d). Compared to the peaks associated with the epoxy groups (c. $1080 \mathrm{~cm}^{-1}$ ) and hydroxyl groups (c. $3400 \mathrm{~cm}^{-1}$ ) in the spectrum of rGO@120, the change in these peak intensities observed for rGO@140 is in accord with a reduction of the epoxy and hydroxyl groups present having occurred in the latter case.

The thermo-voltages measured across the opposite ends of the different "weight ratio" samples (temperature difference $36 \mathrm{~K})$ are presented in Fig. S4 (ESI $\dagger$ ). The maximum voltage (of $1.76 \mathrm{mV}$ ) was observed for rGO@100, with the magnitude of voltage decreasing sharply with increase in reduction temperature. The thermo-voltages obtained for GO/CNT $(1: 0.5)$, $\mathrm{GO} / \mathrm{CNT}(1: 1)$, and $\mathrm{GO} / \mathrm{CNT}(1: 2)$, are $0.0332,0.0445$, and $0.0326 \mathrm{mV}$, respectively. The corresponding Seebeck coefficients are presented in Fig. 2a and Table 1. An exceptionally high Seebeck coefficient of $1350 \mu \mathrm{V} \mathrm{K}{ }^{-1}$ was obtained for rGO@100. However, the reduction of GO at elevated temperature significantly decreases the Seebeck coefficient value. For example, the Seebeck coefficient for rGO@400 is only $6.7 \mu \mathrm{V} \mathrm{K}^{-1}$ while, on the other hand, the value for GO/CNT $(1: 1)$ is $44.5 \mu \mathrm{V} \mathrm{K}^{-1}$. Lower and higher ratios of GO: CNT were both observed to result in somewhat lower Seebeck coefficients. For example, the values for GO/CNT $(1: 0.5)$ and GO/CNT $(1: 2)$ are 32.0 and $28.6 \mu \mathrm{V} \mathrm{K}^{-1}$, respectively.

The electrical conductivities of the samples measured under their respective "temperature-difference" conditions are presented in Fig. $2 \mathrm{~b}$ and Table 1 . The electric conductivities of GO and rGO@100 are both very low but the values increase significantly for the other (higher-temperature reduction) samples. The electrical conductivities increase from $5.98 \times$ $10^{-8} \mathrm{~S} \mathrm{~cm}^{-1}$ for GO to $3.44 \times 10^{-2} \mathrm{~S} \mathrm{~cm}^{-1}$ for rGO@400. Interestingly the electric conductivity of $\mathrm{GO} / \mathrm{CNT}$ is much higher than even rGO@400. Moreover, the value increases with an increasing proportion of CNT in the GO/CNT sample. The highest electric conductivity of $6.52 \times 10^{-1} \mathrm{~S} \mathrm{~cm}^{-1}$ was achieved for GO/CNT $(1: 2)$.

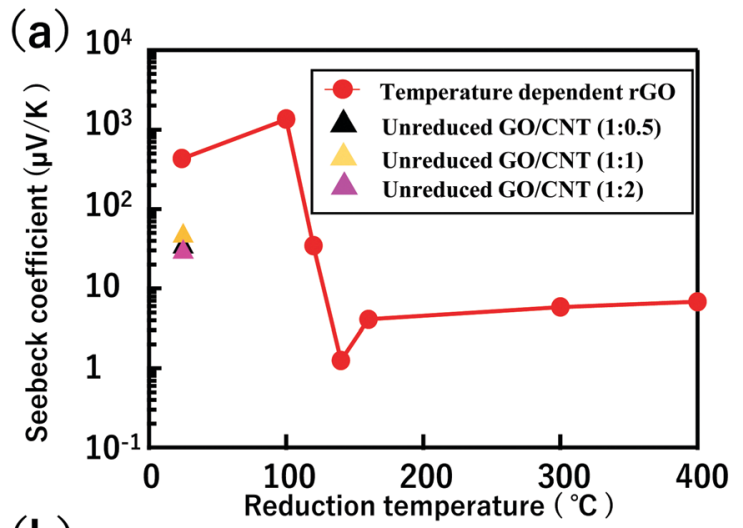

(b)

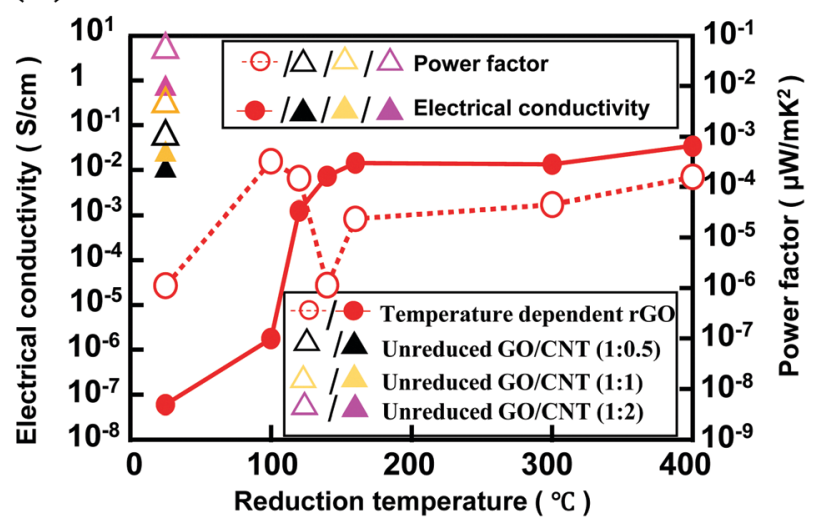

Fig. 2 Thermoelectric properties of GO-hybrids. (a) Seebeck coefficients for $\mathrm{GO}, \mathrm{rGO}\left(100-400^{\circ} \mathrm{C}\right)$ and $\mathrm{GO} / \mathrm{CNT}(1: 0.5,1: 1$, and $1: 2$ weight ratios); (b) electrical conductivities and thermoelectric power factors for GO, rGO $\left(100-400{ }^{\circ} \mathrm{C}\right)$, and GO/CNT $(1: 0.5,1: 1$, and $1: 2)$.

Table 1 Thermoelectric properties of the prepared GO-based hybrid membranes

\begin{tabular}{llll}
\hline & $\begin{array}{l}\text { Seebeck coefficient } \\
\text { conductivity }\left(\mu \mathrm{V} \mathrm{K}^{-1}\right)\end{array}$ & $\begin{array}{l}\text { conductivity } \\
\left(\mathrm{S} \mathrm{cm}^{-1}\right)\end{array}$ & $\begin{array}{l}\text { Power factor } \\
\left(\mu \mathrm{W} \mathrm{mK} \mathrm{mK}^{-2}\right)\end{array}$ \\
\hline GO/CNT $(1: 0.5)$ & 32.0 & $9.85 \mathrm{E}-03$ & $1.01 \mathrm{E}-03$ \\
GO/CNT $(1: 1)$ & 44.5 & $2.23 \mathrm{E}-02$ & $4.41 \mathrm{E}-03$ \\
GO/CNT $(1: 2)$ & 28.6 & $6.52 \mathrm{E}-01$ & $5.33 \mathrm{E}-02$ \\
GO & 432 & $5.96 \mathrm{E}-08$ & $1.11 \mathrm{E}-06$ \\
rGO@100 & 1350 & $1.77 \mathrm{E}-06$ & $3.22 \mathrm{E}-04$ \\
rGO@120 & 34.4 & $1.26 \mathrm{E}-03$ & $1.49 \mathrm{E}-04$ \\
rGO@140 & 1.25 & $7.33 \mathrm{E}-03$ & $1.14 \mathrm{E}-06$ \\
rGO@160 & 4.07 & $1.44 \mathrm{E}-02$ & $2.23 \mathrm{E}-05$ \\
rGO@300 & 5.8 & $1.35 \mathrm{E}-02$ & $5.55 \mathrm{E}-05$ \\
rGO@400 & 6.7 & $3.44 \mathrm{E}-02$ & $1.57 \mathrm{E}-04$
\end{tabular}

Finally, the power factors for all membrane samples were calculated using power factor $=\sigma \times S^{2}$ and are presented in Fig. $2 \mathrm{~b}$ and Table 1.

The maximum power factor obtained for the samples prepared is $5.33 \times 10^{-2} \mu \mathrm{W} \mathrm{mK}{ }^{-2}$ for the GO/CNT $(1: 2)$ product. The power factors for GO/CNT $(1: 0.5)$ and GO/CNT $(1: 1)$ are $1.01 \times 10^{-3}$ and $4.41 \times 10^{-3} \mu \mathrm{W} \mathrm{mK}^{-2}$, respectively. Pristine GO shows a very low power factor of $1.11 \times 10^{-6} \mu \mathrm{W} \mathrm{mK} \mathrm{m}^{-2}$. For the rGO samples, rGO@100 shows the maximum power factor of $3.22 \times 10^{-4} \mu \mathrm{W} \mathrm{mK} \mathrm{m}^{-2}$ (Table 1 ). 


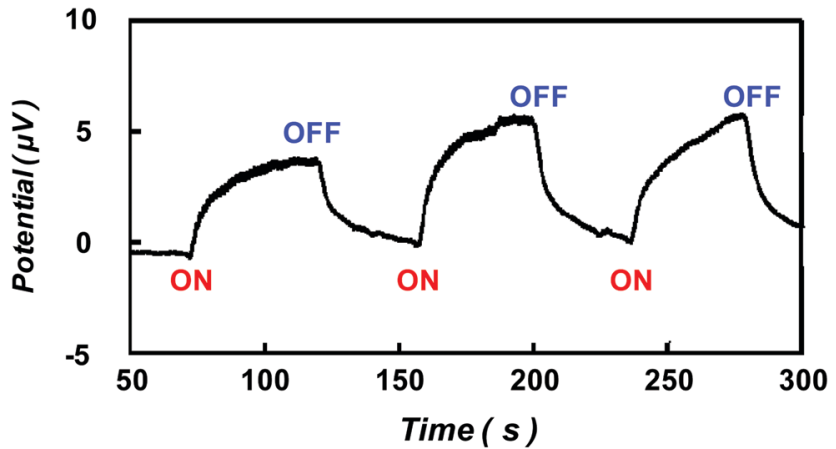

Fig. 3 Thermoelectric response of rGO@100 membrane with temperature difference employed form body heat (temperature difference $12 \mathrm{~K}$ ).

As an extension of the above, we have employed body heat (finger contact) as the heat source to exploit the Seebeck effect in a rGO@100 film. In this case a temperature difference of $12 \mathrm{~K}$ (versus ambient) was achieved. The excellent reversible behaviour following alternate finger contact/non-contact against time is shown In Fig. 3.

In general, the Seebeck coefficient and the electrical conductivity of materials depend on the charge carrier concentration; with an inverse relationship existing between them. ${ }^{25}$ Thus, semiconductors/insulators have large Seebeck coefficients and hence low electrical conductivities. GO and GO@100 have very low carrier concentrations, reflecting the presence of the oxygen-containing functional groups, and hence high Seebeck coefficients and very low electronic conductivity. Compared to the pristine GO, the rGO@100 showed the higher Seebeck coefficient value which might be attributed to the removal of the water molecules. In principle, the adsorbed water molecules are removed from the GO during calcination at $100{ }^{\circ} \mathrm{C}^{26}$ Upon reduction at elevated temperatures, the oxygen-functionalized groups are decomposed (Fig. 1c and d) leading to the restoration of $\mathrm{sp}^{2}$-carbons in the membrane structure and resulting in higher carrier concentrations with a concomitant increase in the electrical conductivity (and accompanied by a decrease in the Seebeck coefficient). ${ }^{25}$

The required properties of an ideal thermoelectric material include (i) an adequate Seebeck coefficient (ii) high electrical conductivity, and (iii) low thermal conductivity. As for graphene, the thermoelectric properties of pristine CNT are limited due to its high thermal conductivity. ${ }^{27}$ In the current study, the synergism arising from employing both these to produce hybrid unreduced GO/CNT membranes has resulted in enhanced thermoelectric properties where CNT contributes to the high electrical conductivity while GO contributes the presence of an adequate Seebeck coefficient. As a consequence, the unreduced GO/CNT (1:2) membrane showed a high thermoelectric power factor of $5.33 \times 10^{-2} \mu \mathrm{W} \mathrm{mK}^{-2}$. Previously there have some reports on rGO and rGO based composites as efficient thermoelectric materials. ${ }^{28-31}$ For example, Mehmood et al. reported the thermoelectric power factor of $2.21 \times 10^{-3} \mu \mathrm{W} \mathrm{K} \mathrm{K}^{-2}$ for flat rGO film. ${ }^{28}$ The power factor of $\sim 0.93 \mu \mathrm{W} \mathrm{K} \mathrm{K}^{-2} \mathrm{~m}^{-1}$ for rGO films obtained from $4 \mathrm{~h}$ hydrothermal reactions and deposited on a glass substrate. ${ }^{29}$ In another study, the power factor value of $\sim 0.43 \mu \mathrm{W} \mathrm{K} \mathrm{K}^{-2} \mathrm{~m}^{-1}$ was reported for rGO with PANI composite material obtained by spark plasma sintering (rGO:PANI as $30: 70) .{ }^{30}$ In addition, the value of pure rGO paper were calculated as $\sim 3.0 \times 10^{-2} \mu \mathrm{W} \mathrm{K} \mathrm{K}^{-2} \mathrm{~m}^{-1}$ which then increase to $1.2 \mu \mathrm{W} \mathrm{mK}{ }^{-2}$ using rGO $+50 \% \mathrm{CNT}$ paper. ${ }^{31}$ However, the current work is the first report for free-standing unreduced $\mathrm{GO}$ / CNT as thermoelectric materials with a comparable power factor of $5.22 \times 10^{-2} \mu \mathrm{W} \mathrm{K} \mathrm{K}^{-2} \mathrm{~m}^{-1}$. Unfortunately, the estimation of the thermal conductivity of our GO/CNT membrane is beyond our current scope. However, some recent studies report the much lower thermal conduction (more than 90\%) of graphene oxide compared to graphene. ${ }^{18,19}$ The oxygen-containing functional groups reduce the efficiency of phonon transport in graphene oxide and adversely affect the thermal performance due to the mean free path of phonons being limited mainly by interior defects resulting from both acoustic mismatch and reduced symmetry in the graphene structure. ${ }^{18,19}$ Arising from this, the resulting unreduced $\mathrm{GO} / \mathrm{CNT}$ composite is expected to exhibit low thermal conductivity and hence shows much promise for lowtemperature driven thermoelectric devices in the future.

\section{Conclusions}

In conclusion, we have successfully prepared freestanding hybrid membranes using unreduced GO and CNT in different ratios which show enhanced thermoelectric properties. In particular, the GO/CNT $(1: 2)$ membrane exhibited a significant enhancement in terms of the Seebeck coefficient, electric conductivity, and thermoelectric power factor with values of

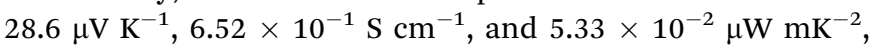
respectively. On the other hand, despite rGO@100 showing a very high Seebeck coefficient, the low electric conductivity reduces its thermoelectric power factor and, consequently, also its suitability for practical application. In addition, at higher reduction temperatures (i.e., that employed to obtain rGO@400), the Seebeck coefficient was sharply reduced resulting in a low power factor. The current study signifies that pristine GO or rGO appear less than ideal for use as thermoelectric materials for practical application. Instead, unreduced GO with electron conductive additives (in the present study CNT) appears a promising pathway towards efficient thermoelectric materials. We propose that the results from the current study will provide a basis for important future developments in the area.

\section{Conflicts of interest}

There are no conflicts to declare.

\section{Notes and references}

1 Z. Soleimanim, S. Zoras, B. Ceranic, S. Shahzad and Y. Cui, Sustainable Energy Technol. Assess., 2020, 37, 100604.

2 P. Ren, Y. Liu, J. He, T. Lv, J. Gao and G. Xu, Inorg. Chem. Front., 2018, 5, 2380. 
3 S. Twaha, J. Zhu, Y. Yan and B. Li, Renewable Sustainable Energy Rev., 2016, 65, 698.

4 B. Poudel, Q. Hao, Y. Ma1, Y. Lan, A. Minnich, B. Yu, X. Yan, D. Wang, A. Muto, D. Vashaee, X. Chen, J. Liu, M. S. Dresselhaus, G. Chen and Z. Ren, Science, 2008, 320, 634-638.

5 R. Venkatasubramanian, E. Siivola, T. Colpitts and B. O'Quinn, Nature, 2001, 413, 597-602.

6 H. J. Goldsmid, Proc. Phys. Soc., London, 1958, 71, 633.

7 B. Russ, A. Glaudell, J. J. Urban, M. L. Chabinyc and R. A. Segalman, Nat. Rev. Mater., 2016, 1, 16050.

8 W. Liu, H. S. Kim, Q. Jie and Z. Ren, Scr. Mater., 2016, 111, 3-9.

9 T. Mori, Small, 2017, 13, 1702013.

10 I. Petsagkourakis, K. Tybrandt, X. Crispin, I. Ohkubo, N. Satoh and T. Mori, Sci. Technol. Adv. Mater., 2018, 19, 836.

11 L. D. Hicks and M. S. Dresselhaus, Phys. Rev. B: Condens. Matter Mater. Phys., 1993, 47, 12727.

12 K. Takahata, Y. Iguchi, D. Tanaka, T. Itoh and I. Terasaki, Phys. Rev. B: Condens. Matter Mater. Phys., 2000, 61, 12551.

13 Y. Mizuguchi, H. Fujihisa, Y. Gotoh, K. Suzuki, H. Usui, K. Kuroki, S. Demura, Y. Takano, H. Izawa and O. Miura, Phys. Rev. B: Condens. Matter Mater. Phys., 2012, 86, 220510.

14 Y. Mizuguchi, S. Demura, K. Deguchi, Y. Takano, H. Fujihisa, Y. Gotoh, H. Izawa and O. Miura, J. Phys. Soc. Jpn., 2012, 81, 114725.

15 Y. Zhang, Q. Zhang and G. Chen, Carbon Energy, 2020, 2, 408.

16 D. Qu, X. Li, H. Wang and G. Chen, Adv. Sci., 2019, 6, 1900584.

17 L. Deng and G. Chen, Nano Energy, 2021, 80, 105448.
18 J. Chen and L. Li, JETP Lett., 2020, 112, 117.

19 X. Mu, X. Wu, T. Zhang, D. B. Go and T. Luo, Sci. Rep., 2014, 4, 3909.

20 W. Wang, Q. Zhang, J. Li, X. Liu, L. Wang, J. Zhu, W. Luo and W. Jiang, RSC Adv., 2015, 5, 8988.

21 D. Narducci, E. Selezneva, G. Cerofolini, S. Frabboni and G. Ottaviani, J. Solid State Chem., 2012, 193, 19.

22 J. Gao, C. Liu, L. Miao, X. Wang, Y. Peng and Y. Chen, RSC Adv., 2016, 6, 31580.

23 O. Okhay, G. Gonçalves, C. Dias, J. Ventura, E. M. F. Vieira, L. M. V. Gonçlves and A. Tkachb, J. Alloys Compd., 2019, 781, 196.

24 H. Im and J. Kim, Carbon, 2012, 50, 5429.

25 J. Choi, N. D. K. Tu, S. S. Lee, H. Lee, H. Kim and J. Kim, Macromol. Res., 2014, 22, 1104-1108.

26 K. Wakata, M. R. Karim, M. S. Islam, R. Ohtani, M. Nakamura, M. Koinuma and S. Hayami, Chem. - Asian J., 2017, 12, 194.

27 N. T. Hung, A. R. T. Nugraha and R. Saito, Energies, 2019, $12,4561$.

28 T. Mehmood, J. H. Kim, D.-J. Lee, S. Dizhur, R. Odessey, E. S. Hirst, R. M. Osgood III, M. H. Sayyad, M. A. Munawar and J. Xu, Mater. Res. Express, 2019, 6, 075614.

29 J. Gao, C. Liu, L. Miao, X. Wang, Y. Peng and Y. Chen, RSC Adv., 2016, 6, 31580.

30 W. Wang, Q. Zhang, J. Li, X. Liu, L. Wang, J. Zhu, W. Luo and W. Jiang, RSC Adv., 2015, 5, 8988.

31 O. Okhaya, G. Gonçalves, C. Dias, J. Ventura, E. M. F. Vieira, L. M. V. Gonçalves and A. Tkach, J. Alloys Compd., 2019, 781, 196. 